\title{
Electron Beam Induced Phase Transformation in Zinc Phosphate
}

\author{
Kun $\mathrm{He}^{1,3}$, Xian Zhang ${ }^{3}$, Yu-Peng Lu ${ }^{3}$, Reza Shahbazian Yassar ${ }^{1,2}$ \\ 1. Department of Materials Science and Engineering, Michigan Technological University, Houghton, \\ Michigan, United States. \\ 2. Department of Mechanical Engineering-Engineering Mechanics, Michigan Technological University, \\ Houghton, Michigan, United States. \\ 3. School of Materials Science and Engineering, Shandong University, Ji'nan, China.
}

Zinc phosphate, which is mainly used to protect metal form corrosion and abrasion, is one of the commonly used biomaterial and industry fields [1]. Herein, we demonstrate that the phase and microstructure of zinc phosphate prepared by phosphating on a stainless steel substrate can be significantly influenced by electron beam. Employing transmission electron microscopy (TEM) and aberration-corrected scanning transmission electron microscopy (STEM) techniques, we obtained unambiguous evidence that the phase and microstructure of zinc phosphate samples changed when the specimens exposed to e-beam. We believe that this condition results in dehydration and eventually the observed microstructure and phase change. In this research, we used two kinds of electron beams to irradiate samples, one was converged beam and another was defocused beam. Our results constitute the first experimental investigation of effect of electron beam on zinc phosphate and the energy transfers from electron beam to sample [2]. In our research, the initial uniform structure changed to a different one with some hollows overlaps each other and some black spots diffusion in the substrate. At the same time, the phase of the sample changed from a kind of amorphous to poly crystalline. Direct evidence of the phase and structure transformation process has advanced our fundamental understanding of microstructure of zinc phosphate coatings, which is of great practical importance for many industrial and medical applications.

\section{References:}

[1] Zhang X., et al, Surface and Coatings Technology 240 (2013), 361-364.

[2] Zheng H., et al, Scientific reports 3 (2013), 1-5. 


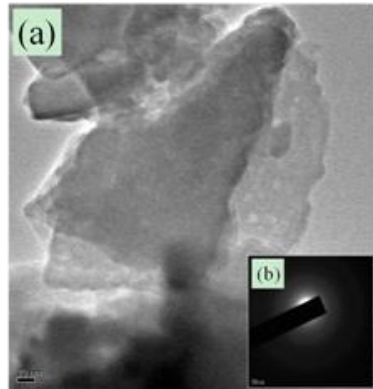

(f)

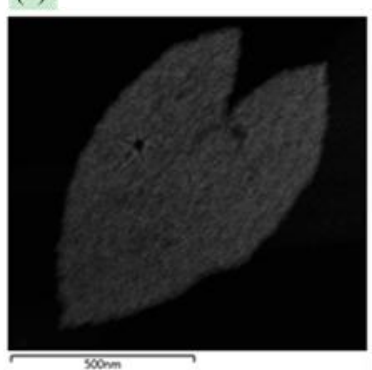

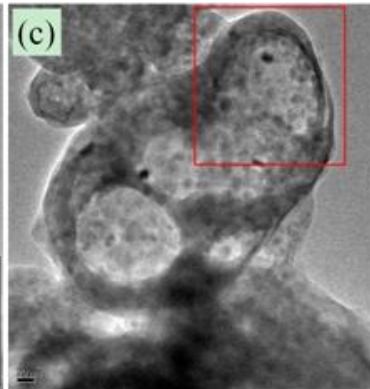

O K series

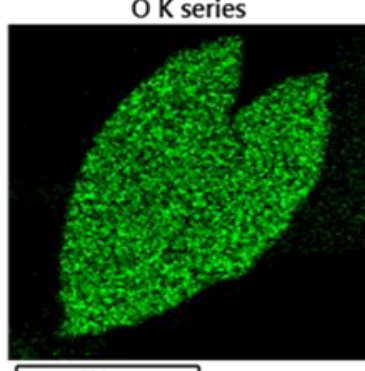

$500 \mathrm{~nm}$

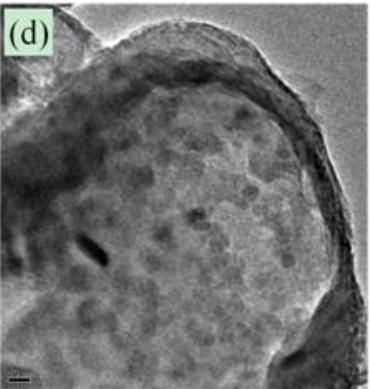

Zn K series
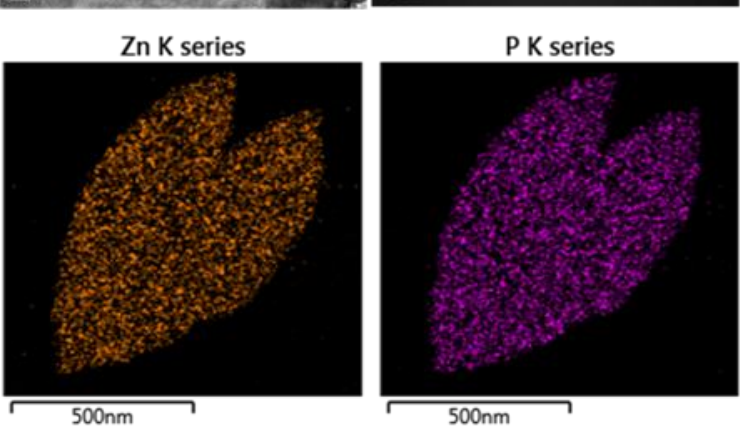

Figure 1. TEM images (diffraction patterns) and EDS mapping of the sample. (a), initial picture; (b), diffraction pattern of initial sample; (c), Samples after electron beam irradiation; (d), magnify image of red square in (c); (e) Diffraction pattern of samples after electron beam irradiation; (f) STEM image and corresponding EDS mapping of sample, with element $\mathrm{O}, \mathrm{Zn}$ and $\mathrm{P}$. 